\title{
Векторный потенциал, эффект Ааронова-Бома и электродинамика в токамаке
}

\author{
Валентин Васильевич Аксенов \\ orcid.org/0000-0001-7525-0619
}

\begin{abstract}
Аннотация В статье показано, что векторный потенциал в электродинамике является вспомогательной функцией, а в некоторых случаях может включать в себя одновременно не силовое магнитное поле. В связи с этим в эффекте Ааронова-Бома обладающая зарядом квантовая частица взаимодействует не с потенциалом, а с не силовым магнитным полем. При этом теорема Стокса, примененная к векторному потенциалу, остается верной и применимой для объяснения эффекта Ааронова-Бома за счет присутствия в векторном потенциале не силового тороидального магнитного поля. Электродинамика в токамаке находит объяснение также в терминах силовых и не силовых магнитных полей.
\end{abstract}

Ключевые слова Электродинамика, векторный потенциал, эффект Ааронова-Бома, электродинамика в токамаке

УДК $550.837 ; 550.37 .382$

\section{1. Введение}

В электродинамике векторный и скалярный потенциалы, описывающие магнитное поле, играют важную роль, являясь объектами пристального изучения [1]. Длительное время в квантовой теории и не только в ней дискуссируется вопрос о физической природе векторного потенциала [1-4].

Наиболее интересный эффект, связанный с векторным потенциалом, зафиксирован в [2], поэтому в физической литературе этот эффект приобрел название эффекта Ааронова-Бома. Отдавая должное достигнутым в [1-4] 
результатам, объясняющим эффект Ааронова-Бома, обратим внимание на тот факт, что при доказательстве физической объективности векторного потенциала в работе [3] допущен отказ от известной теоремы Стокса о циркуляции вектора вдоль замкнутого контура, на основании использования потенциалов нулевого поля Тихонова-Четаева [11]. Известное определение векторного потенциала $A$ позволяет записать: $\nabla \cdot H=0, \quad \mathrm{H}=\nabla \times A$, где $H$ - магнитное поле. Когда $H=\nabla \times A=0$, то векторный потенциал сохраняется из-за $A=\nabla \varphi$, где $\varphi$ - произвольная скалярная функция, названная в [11] потенциалом нулевого поля. Это обстоятельство позволило в [1-4] считать, что в эксперименте Ааронова-Бома квантовая частица взаимодействует с векторным потенциалом, поскольку он сохраняется при $\nabla \times A=0$.

Однако, на основании теоремы Стокса, примененной к векторному потенциалу $A$ можно показать, что в эксперименте Ааронова-Бома вне бесконечно-длинного соленоида нет не только магнитного поля $H$, но в стандартном определении и векторного потенциала $A$. Действительно, по теореме Стокса:

$$
\int_{L}(A \cdot d l)=\int_{S}(\nabla \times A \cdot d s)=\left.\int_{S}(H \cdot d s)\right|_{H=0}=0,
$$

в точках вне соленоида магнитное поле равно нулю $H=0$. Оно сосредоточено внутри соленоида. В тех же точках, где $H=0$, вектор-потенциал также равен нулю, так как интегрирование в первом интеграле выше приведенной формулы, по контуру $L$, не охватывающему соленоид, при стягивании в точку дает нуль из-за $H=0$ в каждой точке. Контура, охватывающие соленоид, дают нуль в первом интеграле этой формулы из-за равенства нулю вектора $A$ (левый интеграл в выше приведенной формуле). Это обстоятельство заметили в [3], поэтому предложили отказаться от теоремы Стокса, путем введения потенциала нулевого поля Тихонова-Четаева [11], который, как показано выше, присутствует, но не дает магнитного поля.

C нашей точки зрения, отказ от теоремы Стокса в этом эксперименте является не оправданным, поэтому его необходимо преодолеть, предложив при этом простой способ экспериментальной проверки полученного в статье результата.

\section{2. Однородная проводящая магнитная среда}

Векторный потенциал $A$ для бездивергентных магнитньх полей в однородной проводящей и магнитной среде вводится стандартным образом:

$$
H=\nabla \times A .
$$


Далее воспользуемся следующим нестандартным определением векторного потенциала, а именно ортогональным разложением векторного потенциала $A$ относительно произвольного вектора $r$ из [12]:

$$
A=(Q r)+\nabla \times(Q r) .
$$

Здесь $Q$ математическая скалярная функция трех переменных класса $C^{\infty}$. Ортогональность такого разложения очевидна. Действительно, например, в сферической системе координат $(r, \theta, \varphi)$, которая используется и в дальнейшем, скалярное произведение слагаемых из (2) есть:

$$
\left((0,0,(Q r)) \cdot\left(\nabla_{\theta}(Q r), \nabla_{\varphi}(Q r), 0\right)\right) \equiv 0 .
$$

Ортогональное разложение (2) разбивает вектор магнитного поля на два слагаемых [12]:

$$
H=\nabla \times(Q r)+\nabla \times \nabla \times(Q r),
$$

каждое из которых представляет известные из [5] магнитные поля: $H_{T}=\nabla \times(Q r) \quad[-, \mathrm{A}, \mathrm{A} /<]-$ тороидальное магнитное поле, $H_{P}=$ $\nabla \times \nabla \times(Q r) \quad[-, \mathrm{A}, \mathrm{A} /<]-$ полоидальное магнитное поле. Размерности введенных магнитных полей определяются размерностью исходного магнитного поля в левой части формулы (4). Справа в (4) располагаются дифференциальные операторы и математические функции изначально размерностью не обладающие. Константы, входящие в функцию $Q$ и обладающие размерностью магнитного поля при дифференцировании размерности не теряют.

Важнейшим свойством разложения магнитного поля (4) является то обстоятельство, что каждое из определенных в (4) магнитных полей имеет свои существенные и особенные свойства. Вихри тороидального магнитного поля порождают полоидальное магнитное поле в любой среде. Действительно:

$$
\nabla \times H_{T}=\nabla \times \nabla \times(Q r)=H_{P}
$$

А вихри полоидального магнитного поля порождают тороидальное только в проводящей и магнитной среде следующим образом:

$$
\nabla \times H_{P}=\nabla \times \nabla \times \nabla \times(Q r)=-\nabla \times(\Delta Q \cdot r)=\chi H_{T} .
$$

Здесь: $\chi$ - константа в однородной проводящей среде, определяемая из соотношений:

$$
\Delta Q=-\chi Q=\left\{\begin{array}{lc}
-\frac{\gamma}{\eta} Q & t=0 \\
-(i \omega \mu \sigma)^{1 / 2} Q & t>0 .
\end{array}\right.
$$


Где $Q=Q(r, \theta, \varphi)$ для $t=0, Q=Q(r, \theta, \varphi) e^{i \omega t}$ для $t>0, Q \in C^{\infty}, \eta=$ $\frac{1}{\sigma \mu}\left[<^{2} / A\right]$ - магнитная вязкость, $\omega[1 / c]-$ круговая частота, $\sigma\left[(><<)^{-1}\right]-$ удельная проводимость, $\mu[3=/<]$ - магнитная проницаемость, $\gamma[</ A]-$ скорость диффузии магнитного поля. Размерность коэффициента $\chi[1 /<]$ - в обеих случаях в (7).

Из (4-6) однозначно вытекают два принципиальных свойства введенных выше магнитных полей.

Тороидальное магнитное поле всюду не силовое в том смысле, что сила Лоренца в его источнике равна нулю. Действительно:

$$
F=\left[\chi H_{T} \times \mu H_{T}\right]=0 .
$$

Равенство нулю векторного произведения в (8) обеспечивает параллельность вектора $H_{T}$ самому себе. В непроводящей среде сила Лоренца $F$ в $(8)$ также равна нулю из-за $\chi=0$. Поэтому будем называть тороидальное магнитное поле $H_{T}$ не силовым магнитным полем.

Полоидальное поле имеет силовой характер. По определению из [5] сила Лоренца в источнике не равна нулю.

$$
F=[\sigma E \times \mu H] \neq 0 .
$$

В непроводящей среде $F=0$ в (9) из-за равенства нулю проводимости $\sigma$. Тем не менее, будем называть полоидальное поле $H_{P}$ силовым магнитным полем. Другое важное свойство состоит в том, что вихри тороидального магнитного поля порождают полоидальное в любой среде (5), в то время как вихри полоидального магнитного поля порождают тороидальное только в проводящей среде (6). Так что их взаимная самогенерация возможна только в проводящей среде.

Эти последние свойства существенны для понимания эффекта АароноваБома и электродинамики в токамаке, с одной стороны, и весьма важны для сохранения теоремы Стокса при объяснении выше названного эффекта Ааронова-Бома.

Формулы (2) и (4) указывают на некоторый дуализм в определении векторного потенциала в том смысле, что если в (2) $\nabla \times(Q r)=H_{T} \neq 0$, то векторный потенциал содержит в себе несиловое магнитное поле и поэтому является явным физическим объектом. Если $H_{T}=0$, то векторный потенциал представлен произвольной скалярной функцией $Q$ и является сугубо математической вспомогательной функцией. Формальный дуализм в определении векторного потенциала содержит в себе принципиальное физическое начало, связанное с известным квантовым эффектом Ааронова-Бома 
[2] с одной стороны, и с существованием так называемых «теорем» антидинамо [7-9], в которых утверждается отсутствие тороидального магнитного поля $H_{T}$ при планальной и цилиндрической симметриях полей и потоков, с другой.

Если $H_{T}$ присутствует в векторном потенциале, то квантовая частица в эффекте Ааронова-Бома испытывает взаимодействие не с математической функцией, а с не силовым тороидальным магнитным полем. При этом теорема Стокса остается справедливой. Действительно:

$$
\oint(A \cdot d l)=\oint(\nabla \times A \cdot d s)=\oint\left(H_{T} \cdot d s\right)+\oint\left(H_{P} \cdot d s\right)=\oint\left(H_{T} \cdot d s\right)+\oint\left(\nabla \times H_{T} \cdot d s\right) .
$$

Соотношения (10) получены с учетом формул (4-5).

Пусть теперь $\nabla \times A=0$, тогда $\nabla \times[(Q r)+\nabla \times(Q r)]=0$. Отсюда:

$$
\nabla \psi=[(Q r)+\nabla \times(Q r)]
$$

Это то же самое, что:

$$
H_{T}=\nabla \times(Q r)=[\nabla \psi-(Q r)]
$$

Здесь $\psi$ и $Q-$ произвольные скалярные функции класса $C^{\infty}$.

Пусть теперь $\nabla \times H_{T}=H_{P}=0$. В этом случае формулу (10) можно переписать следующим образом:

$$
\oint(A \cdot d l)=\oint(\nabla \times A \cdot d s)=\oint\left(H_{T} \cdot d s\right)=\oint([\nabla \psi-(Q r)] \cdot d s) \neq 0 .
$$

Из сказанного выше следует, что равенство $\nabla \times A=0$, вследствие формулы (2), приводит к равенству нулю только силовой части магнитного поля $H_{P} . \mathrm{B}$ то время как интеграл от несилового поля $H_{T}$ в $(10)$ остается не равным нулю в связи с изначально отличными от нуля скалярными функциями $\psi$ и $Q$. Это обстоятельство сохраняет действие теоремы Стокса с одной стороны, и подтверждает дуализм в определении векторного потенциала, - с другой.

\section{3. Бесконечно длинный цилиндрический соленоид с электриче-} ским током (эффект Ааронова-Бома)

Пусть в пространстве $\Re^{3}$ вдоль координаты $z$ прямоугольной системы координат $x, y, z$ расположена бесконечно длинная цилиндрическая катушка (соленоид) с плотной намоткой, в центре цилиндра которой закреплена, кроме прямоугольной, еще и сферическая система координат $(r, \theta, \varphi)$. В проводе катушки циркулирует постоянный электрический ток с плотностью $J$ 
или переменный с плотностью $J \cdot e^{i \omega t}$. Согласно определению (1) и стандартным уравнениям Максвелла эти токи связаны с векторным потенциалом уравнениями $\nabla \nabla \cdot A-\nabla \times \nabla \times A=J$ или $\nabla \nabla \cdot A-\nabla \times \nabla \times A+\chi^{2} A=$ $J, 345 \chi=(\mathrm{i} \omega \mu \sigma)^{1 / 2}$. Множитель $e^{i \omega t}$ в последнем уравнении опущен. Ясно, что в катушке циркулирует только $\varphi$-товая компонента плотности тока $J_{\varphi}$. Спроектируем первое уравнение на $\varphi$-товую ось сферической системы координат:

$$
\begin{gathered}
l \frac{1}{r \sin \theta} \frac{\partial}{\partial \theta} \sin \theta \frac{\partial A_{\varphi}}{\partial \varphi}+\frac{1}{r^{2} \sin ^{2} \theta} \frac{\partial^{2} A_{\varphi}}{\partial \varphi^{2}}+\frac{1}{r} \frac{\partial^{2} r A_{\varphi}}{\partial r^{2}}+\frac{\cos \theta}{r^{2} \sin ^{2} \theta} \frac{\partial A_{\theta}}{\partial \varphi}- \\
-\frac{\cos \theta}{r^{2} \sin \theta} \frac{\partial A_{\varphi}}{\partial \varphi}+\frac{1}{r^{2}} \frac{\partial}{\partial \theta} \frac{1}{\sin \theta} \frac{\partial}{\partial \theta} \sin \theta A_{\varphi}-\frac{1}{r} \frac{\partial^{2} A_{\varphi}}{\partial \theta \partial \varphi}+\frac{2}{r^{2} \sin \theta} \frac{\partial A_{r}}{\partial \varphi}=J_{\varphi}
\end{gathered}
$$

Если спроектировать второе уравнение, то к (13) добавится еще слагаемое $\chi^{2} A_{\varphi}$. Нетрудно заметить, что, согласно определению (2), последнее слагаемое в (13) есть как раз деленная на координату $r$ удвоенная напря-

женность $H_{T \theta}$ - компоненты несилового магнитного поля $H_{T}\left(\frac{2}{r^{2} \sin \theta} \frac{\partial A_{r}}{\partial \varphi}=\right.$ $\left.\frac{2}{r \sin \theta} \frac{\partial Q}{\partial \varphi}=\frac{2}{r} H_{T \theta}\right)$. Поэтому выражение (13) есть прямое доказательство генерирования током проводимости $\left(J_{\varphi}-\right.$ компонентой $)$ не силовой компоненты магнитного поля $H_{T \theta}$, которая, согласно формуле $(12)$, остается в пространстве $\Re^{3}$ даже при $\nabla \times A=0$.

Экспериментальную проверку изложенного здесь можно сделать, измерив магнитометром, использующим пробный чувствительный магнитик, закрепленный на жесткой тонкой кварцевой нити (аналог магнитной стрелки компаса), непосредственно напряженность тороидального магнитного поля вблизи плотно намотанной длинной катушки с током.

\section{4. Электродинамика в токамаке}

Выявленный выше эффект существенен в совсем казалось бы другом эксперименте, а именно в магнитном поле токамака. Если катушку с током из эксперимента [2] соединить в «бублик», то образуется тороидальная катушка, которая присутствует в установке для термоядерного синтеза [13]. Взаимодействие определенных здесь с помощью (4-6) магнитных полей: продольного тороидального в токамаке и образованного внутри токамака током кольцевого проводника полоидального [13], может способствовать появлению неустойчивости плазменного шнура внутри тороидальной катушки за счет так называемого динамо-возбуждения магнитного поля [5]. Другими словами за счет эффекта самовозбуждения, согласно (5) и (6), тороидаль- 
ным полем полоидального и наоборот при появлении внутри токамака проводящего плазменного шнура.

Действительно, согласно [13], магнитное поле в токамаке создается двумя типами источников: тороидальное магнитное поле создается обмотками тороида, полоидальное - внутренним проводником с током.

Согласно определениям (4-6) и результатам из [12], электродинамика магнитного поля внутри токамака, скорее всего, подчиняется следующим уравнениям:

$$
H_{T}=\nabla \times(Q r), \quad \mathrm{H}_{P}=\nabla \times \nabla \times(Q r), \quad \nabla \times H_{T}=H_{P}, \quad \nabla \times H_{P}=\chi H_{T},
$$

где $\gamma$ - скорость диффузии поля в плазме тора, $\mu$ - магнитная проницаемость в плазме, $\sigma$ - проводимость плазмы в пространстве тора. Согласно (14), вихри несилового тороидального магнитного поля создают силовое полоидальное магнитное поле и наоборот. Это есть один из вариантов, так называемого, динамо-возбуждения магнитного поля [5]. Чем напряженнее тороидальное магнитное поле, создаваемое обмотками тороида, а оно в токамаке иногда достигает 3-5 Тл [13], тем интенсивнее будет созданное им дополнительное полоидальное магнитное поле $H_{P}$. Фактически происходит самовозбуждение и бесконтрольное нарастание магнитного поля различной топологии за счет самого магнитного поля, находящегося внутри токамака и при появлении внутри токамака проводящей плазмы. Это приведет к появлению неконтролируемой неустойчивости плазменного шнура. Оценка самовозбуждения в большой модели МТР из [13], согласно определениям из (4-6), выглядит следующим образом. Если принять $\nabla \times \sim \frac{1}{L}$, где $L-$ линейный размер плазменного шнура внутри токамака, то:

$$
\frac{1}{L} H_{P}=\frac{\gamma}{\eta} H_{T}, \quad \frac{1}{L} H_{T}=H_{P} .
$$

Пусть малый радиус плазменного шнура $R=2$ м, тогда $L=2 \pi R=4 \pi$ м, а напряженность $\left|H_{T}\right|=5$ Тл. Тогда возбуждаемая тороидальным магнитным полем напряженность дополнительного полоидального магнитного поля будет порядка:

$$
\left|H_{P}^{\prime}\right|=5 / 4 \pi \approx 0,4
$$

При этом оценка скорости диффузии по первоначальным магнитным полям следующая:

$$
\gamma=\frac{\eta}{L} \frac{\left|H_{P}\right|}{\left|H_{T}\right|}
$$


В свою очередь дополнительное тороидальное магнитное поле будет прирастать на величину:

$$
H_{T}^{\prime}=\frac{\eta}{L \gamma} H_{P}^{\prime}=\frac{H_{T}}{H_{P}} H_{P}^{\prime}=\frac{2}{H_{P}} .
$$

Процесс самовозбуждения будет нарастать за счет взаимной генерации упомянутых магнитных полей. При этом с ростом температуры внутри токамака скорость диффузии также будет нарастать за счет падения проводимости в плазменном шнуре и росту полоидального поля внутри токамака.

Естественно, что сформулированный здесь подход к описанию электродинамики в токамаке нуждается в более тщательном анализе с привлечением уравнения Больцмана, описывающего поведение частиц плазмы при возрастании температуры в таком сложном магнитном поле, возникающем внутри токамака. В настоящее время электродинамика в токамаке описывается известными классическими уравнениями Максвелла, которые несколько отличаются по своей сути от уравнений (4-6).

\section{5. Заключение}

C нашей точки зрения, квантовый эффект Ааронова-Бома вызван все тем же магнитным полем, только его несиловой составляющей. При этом теорема Стокса остается верной и в этом случае. Дуализм векторного потенциала $A$ в его нестандартном определении из [12] с помощью формул (2) полностью подтверждается эффектом Ааронова-Бома.

Что касается электродинамики в токамаке, то за счет самогенерации здесь определенных тороидальных и полоидальных магнитных полей в проводящей среде внутри токамака может возникнуть неуправляемая неустойчивость в плазменном шнуре, которую следует предотвращать. Как это делать есть другая более фундаментальная задача, может быть вне технологии токамака.

\section{Список литературы}

1. Jain A.K. Data clustering: a review [Text] / A.K. Jain, M.N. Murty, P.J. Flynn // ACM Computing Surveys/ - Vol. 31. - No. 3. - P. 264-323.

2. Бериков В.С. Современные тенденции в кластерном анализе [Текст] / В.С. Бериков, Г.С. Лбов // Всероссийский конкурсный отбор обзорно-аналитических статей по приоритетному направлению «Информационно-телекоммуникационные системы». 2008. $-26 \mathrm{c}$.

3. Данилова О.Т. Приближенный глобальный алгоритм решения задачи коммивояжера с неравенством треугольника [Текст] / О.Т. Данилова, Р.Т. Файзуллин // Математические структуры и моделирование : [сб. науч. тр.] / Ом. гос. ун-т. - Омск, 2003.

- Вып. 11. - С. 5-9. 
4. Файзуллин Р.Т. Гладкие приближения в задаче коммивояжёра [Текст] / Р.Т. Файзуллин, Р.Р. Файзуллин // Таврический вестник информатики и математики. - 2004. - №27. - С.72-76.

\section{Валентин Васильевич Аксенов orcid.org/0000-0001-7525-0619}

Институт вычислительной математики и математической геофизики $\mathrm{CO}$ РАН, Россия

E-mail:Aksenov@omzg.sscc.ru

\section{Valentin V. Aksenov}

\section{Vector Potential, Aharonov-Bohm Effect and Electrodynamics of Tokamac}

This paper proves that a vector potential in Electrodynamics is both an auxiliary function and a non-force magnetic field. In this connection in the AharonovBohm effect, a quantum particle interacts with a non-force magnetic field. The Stokes theorem remains valid and applicable to explanation of the AharonovBohm effect and to the electrodynamics of tokamac. Electrodynamics in a tokamac can be explained in terms of power and magnetic force fields.

Keywords Electrodynamics, vector potential, Aharonov-Bohm effect, Tokamac 